\title{
Contexte actuel et perspectives du secteur pétrolier
}

Nathalie ALAZARD-TOUX

IFP

Direction des études économiques

$1 \& 4$ avenue de Bois-Préau,

92852 Rueil-Malmaison Cedex

Fax : 0147527082

<nathalie.alazard@ifp.fr>

\section{Une perte de flexibilité}

Le contexte pétrolier actuel se caractérise par une perte de flexibilité.

Les années 70 avaient créé des surcapacités sur l'ensemble de la chaîne pétrolière aussi bien au niveau de la production qu'au niveau du raffinage. Aujourd'hui, ces surcapacités ont pratiquement disparu et le secteur fonctionne en flux tendus (figure 1).

\begin{abstract}
The tripling of oil prices during the last four years is the consequence of a lost of flexibility all along the oil supply chain. The surplus capacities of production as well as refining created by the previous oil shocks have disappeared. The fundamentals of oil market show that, unless a sharp slowdown of world oil demand, the present lack of flexibility will continue in the short to medium term. The price of the barrel of oil should remain at a high level in the coming years, furthering the development of alternative solutions to oil products as for example biofuels in transportation sector.
\end{abstract}

Key words: biofuels, oil market evolution, transportation sector

Les capacités excédentaires de production situées au niveau des pays de l'Opep, ces capacités dormantes qui, jusqu'à présent, pouvaient être mobilisées en cas de ruptures locales et temporaires d'approvisionnement ont fortement diminué. L'augmentation de la demande pétrolière mondiale s'est accélérée au début des années 2000 devenant bien supérieure à l'accroissement moyen observé dans les années 90 qui était de l'ordre de 1,5\% voire légèrement inférieur. En 2003, l'accroissement de la demande a été de plus de $2 \%$. En 2004, la croissance a dépassé $3 \%$. Cette accélération de la demande est due à la Chine, en grande partie, mais de manière plus générale à l'ensemble des régions du globe.

Dans le même temps, la production a continué à augmenter mais absolument pas dans les mêmes proportions. Principale conséquence de ceci, les capacités excédentaires de produc-

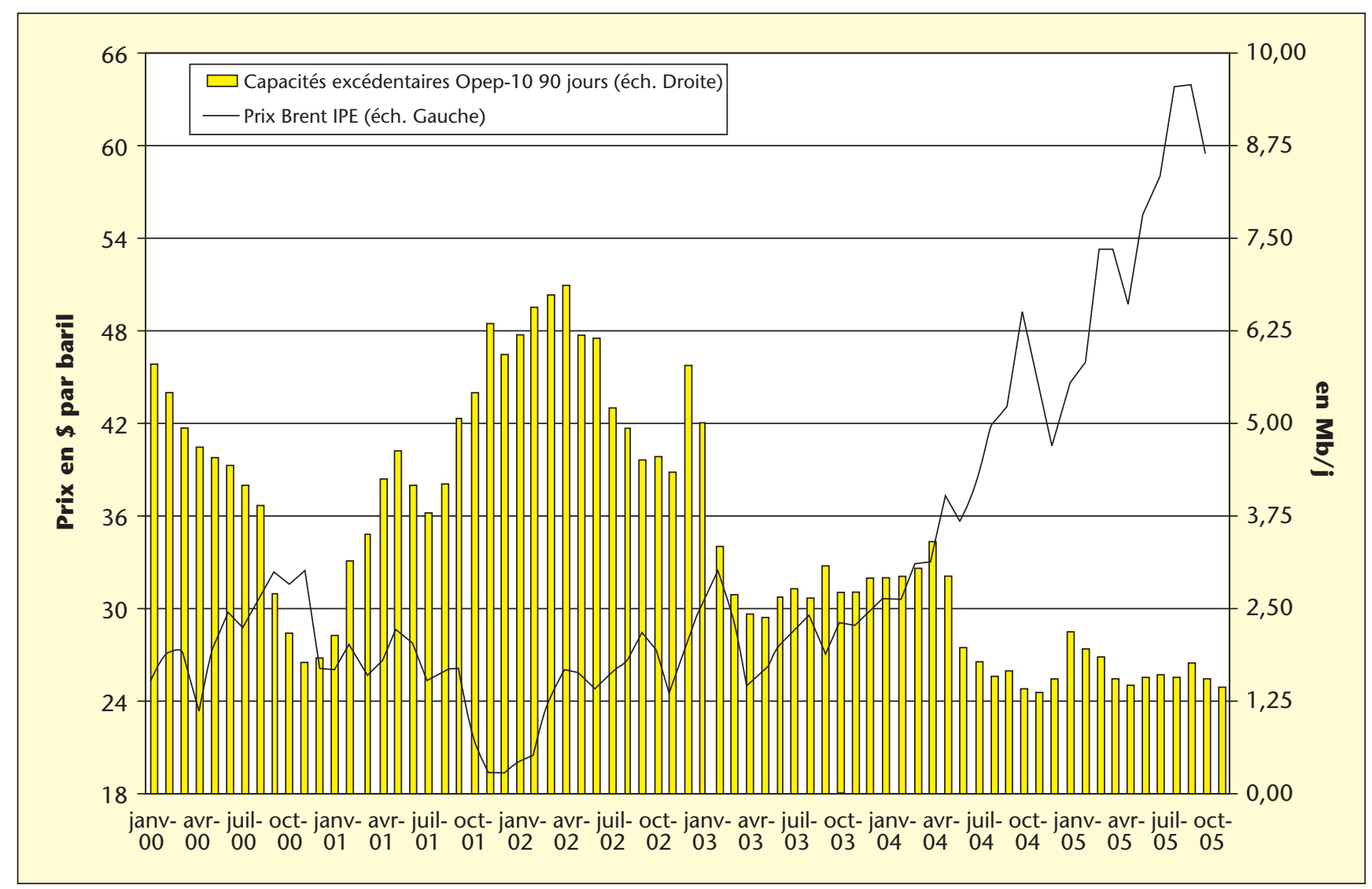

Figure 1. Les capacités de production résiduelles de l'Opep sur les derniers mois.

Source: AIE-PLATTS-IFP. 


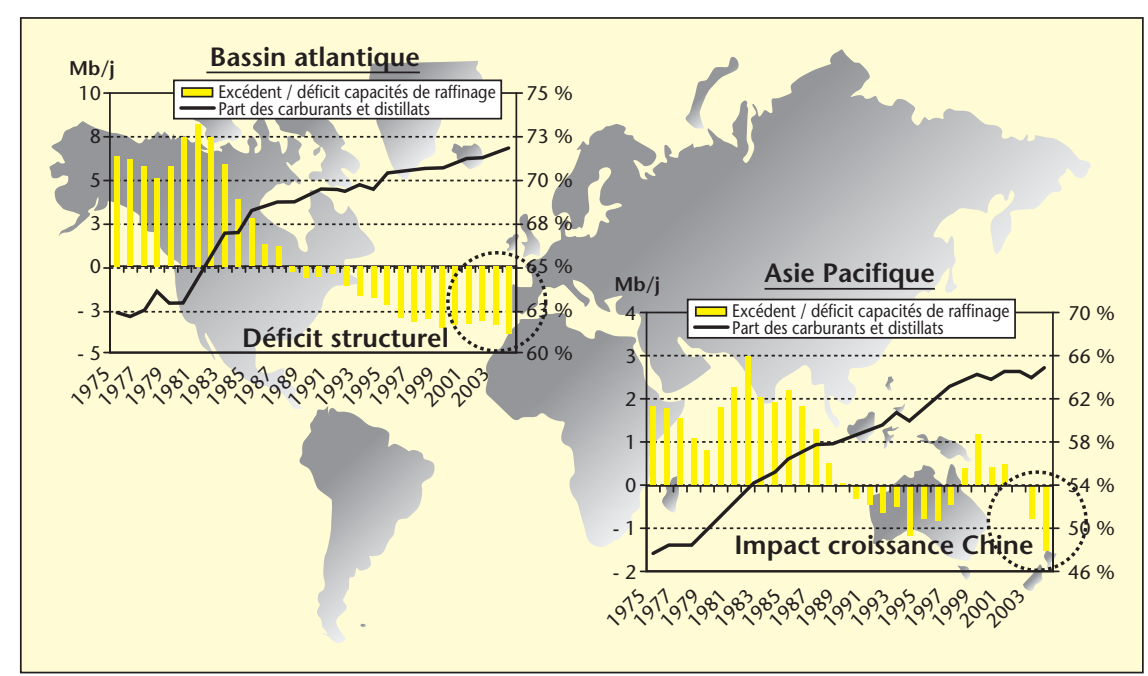

Figure 2. Un manque de capacités de raffinage sur le Bassin atlantique et la zone Asie-Pacifique. Source : AIE-BIP-IFP.

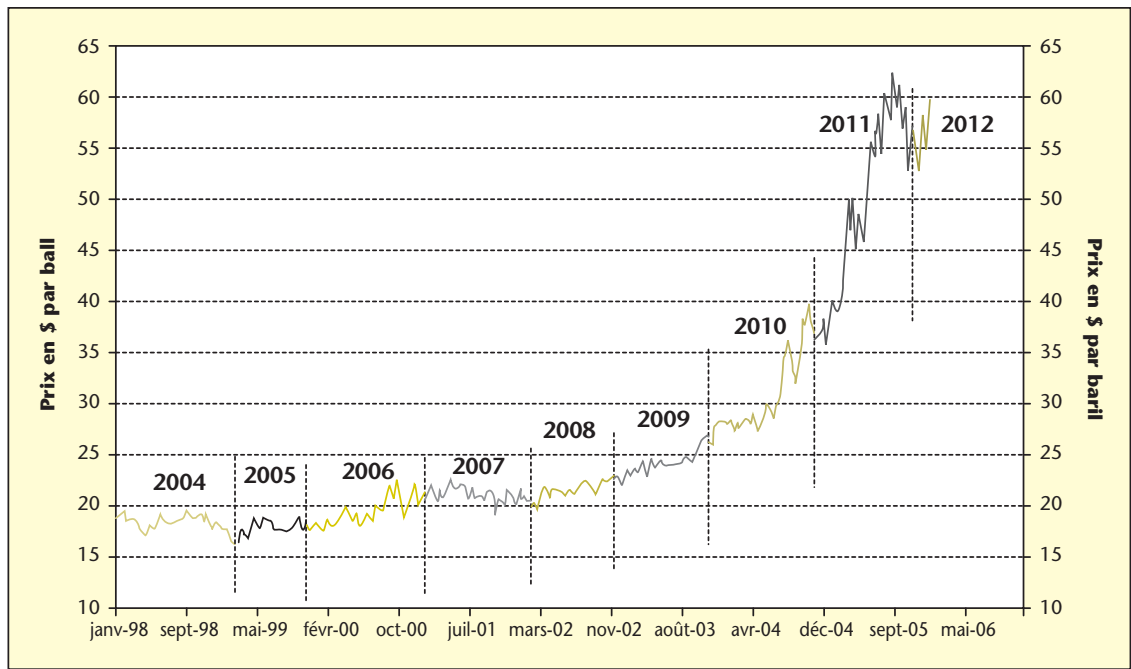

Figure 3. Une nouvelle ère de prix du pétrole. Évolution de la cotation à long terme (7 ans Nymex). Source: Nymex-IFP.

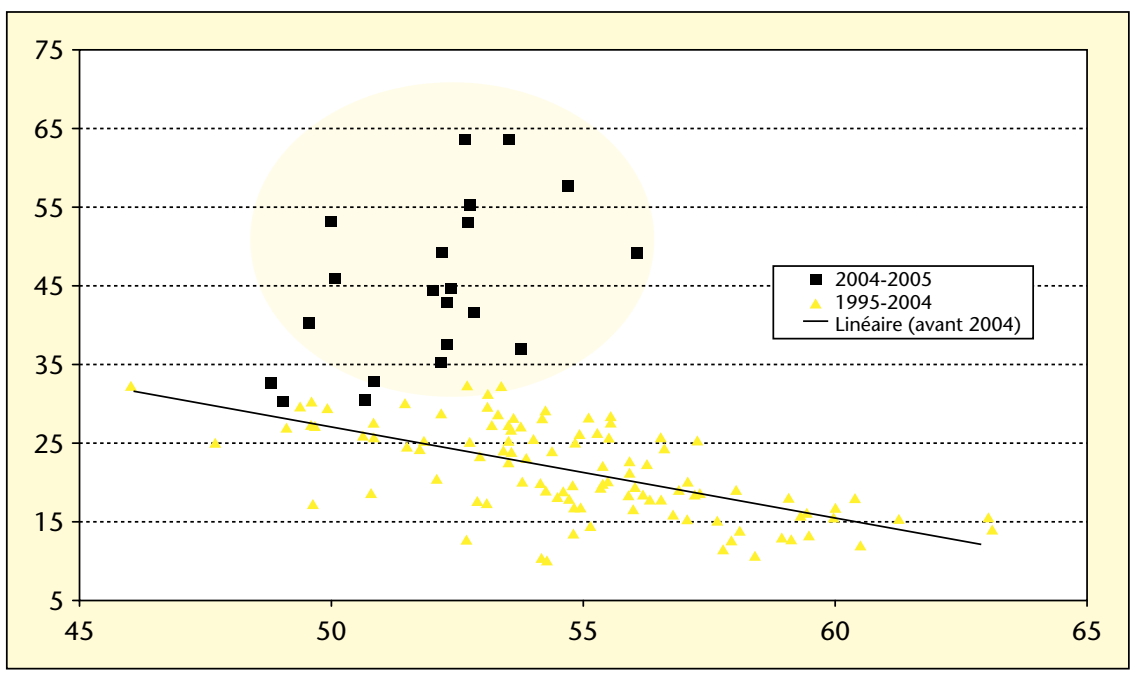

Figure 4. Les relations stock OCDE-prix du brut : rupture à partir de 2004. Source: IFP. tion ont été très fortement érodées. Alors qu'on fonctionnait dans les années 90 avec des capacités excédentaires de production qui représentaient plus de $5 \%$ de la consommation mondiale en moyenne, le secteur disposait en 2005 de capacités excédentaires de production qui représentent moins de $2 \%$ de la consommation mondiale.

Le deuxième niveau de perte de flexibilité concerne l'outil de raffinage. Comme pour les capacités de production, les deux chocs pétroliers des années 70 avaient généré des surcapacités. Ces surcapacités ont peu à peu diminué tout au long des années 90. Aujourd'hui, le taux d'utilisation de l'outil de raffinage mondial est proche de $90 \%$. Les capacités de distillation sont même dans certaines zones, et notamment les principales zones de consommation (Amérique du Nord, Asie), totalement insuffisantes (figure 2).

Au-delà du problème quantitatif, existe un problème qualitatif d'adaptation de l'outil de raffinage au type de produits demandés. Le poids des carburants et, de manière plus générale, des produits légers dans la demande mondiale $s^{\prime}$ est fortement accru au cours des dernières décennies. Dans le même temps, les capacités de conversion qui permettent la production de produits légers à partir de bruts même assez lourds ont augmenté de manière insuffisante. Cette insuffisance des capacités de conversion devient aujourd'hui plus aiguë avec la mobilisation des capacités résiduelles de production de l'Opep, constituées d'une part importante de bruts lourds et soufrés.

On se trouve alors dans une situation où l'insuffisance des capacités de conversion devient un véritable goulet d'étranglement au-delà de la capacité de distillation qui elle-même est tout juste satisfaisante.

\section{Le marché a un peu perdu ses repères}

Le prix d'équilibre, qui représente la perception qu'ont les acteurs du marché, de la manière dont le prix devrait évoluer sur le long terme, s'est au cours de ces trois dernières années profondément modifié (figure 3).

La saturation des capacités de production et de raffinage s'est traduite par une forte hausse du prix du brut sur les marchés à terme pour livraison à plusieurs années (l'échéance la plus lointaine traitée actuellement étant 2012). La cotation du brut à 7 ans qui fluctuait autour de 15-25 \$/b jusqu'en 2002 croît régulièrement depuis 2003 pour s'établir aujourd'hui au-dessus de $60 \$ / b$. Le marché est aujourd'hui convaincu que même sous l'hypothèse d'une croissance modérée de la demande, les tensions apparues en 2004 perdureront plusieurs années. Les inquiétudes sur l'aptitude des pays 
producteurs non-membres de l'Opep à accrô̂tre à court/moyen terme leur production et renouveler leurs réserves sont fortes.

Avec un prix d'équilibre qui restait relativement stable à environ $20 \$ / b$, les variations du baril autour du prix d'équilibre étaient, par le passé, liées au niveau des stocks industriels dans les pays de l'OCDE (exprimé en jours de couverture de la consommation). L'élévation rapide du prix d'équilibre, matérialisé par le prix du baril à long terme, a contribué à rompre la relation entre le prix et le niveau des stocks (figure 4). Depuis 2004, les statistiques de stocks, concernant notamment le marché américain, ne génèrent que des variations d'autant plus faibles que le prix d'équilibre est élevé.

\section{Que peut-on attendre à court/moyen terme ?}

Quelle évolution des équilibres et du prix du baril ?

Les investissements en cours ou à venir vont-ils permettre de desserrer les contraintes?

L'observation du passé montre que toute augmentation ou baisse du prix moyen annuel du baril est suivie d'une hausse ou d'une baisse des investissements en exploration-production. Aujourd'hui, les investissements en explorationproduction augmentent, le problème est qu'ils n'augmentent pas dans des proportions suffisantes.

Premièrement, les domaines miniers les plus intéressants, ceux dont le potentiel géologique est fort, ne sont pas forcément ouverts aux investisseurs privés que sont les grandes compagnies pétrolières internationales : des pays restent fermés comme l'Arabie Saoudite et le Mexique. L'exploitation de la ressource demeure opérée par la compagnie nationale. Dans d'autres pays théoriquement ouverts, investir est en pratique très difficile comme en Russie ou en Irak (problèmes de sécurité, modifications fréquentes des conditions contractuelles, obstacles divers aux investissements privés, etc.).

Autre frein, le secteur se heurte aujourd'hui au problème de la saturation des moyens humains et techniques. À titre d'illustration, explorer et produire du pétrole nécessite de pouvoir faire des forages. Or, aujourd'hui en offshore, la demande en appareils de forage est telle que ces appareils sont réservés plusieurs mois à I'avance (voire 1 ou 2 ans à l'avance) et que les taux de location de ces appareils explosent (figure 5). Ceci signifie aussi que l'accroissement des investissements ne se traduit pas par un accroissement équivalent de l'activité, une partie des investissements additionnels étant absorbée par la hausse des coûts.

Pour finir, il y a une certaine crainte des compagnies nationales à accroître trop rapidement leurs capacités de production. Elles n'oublient

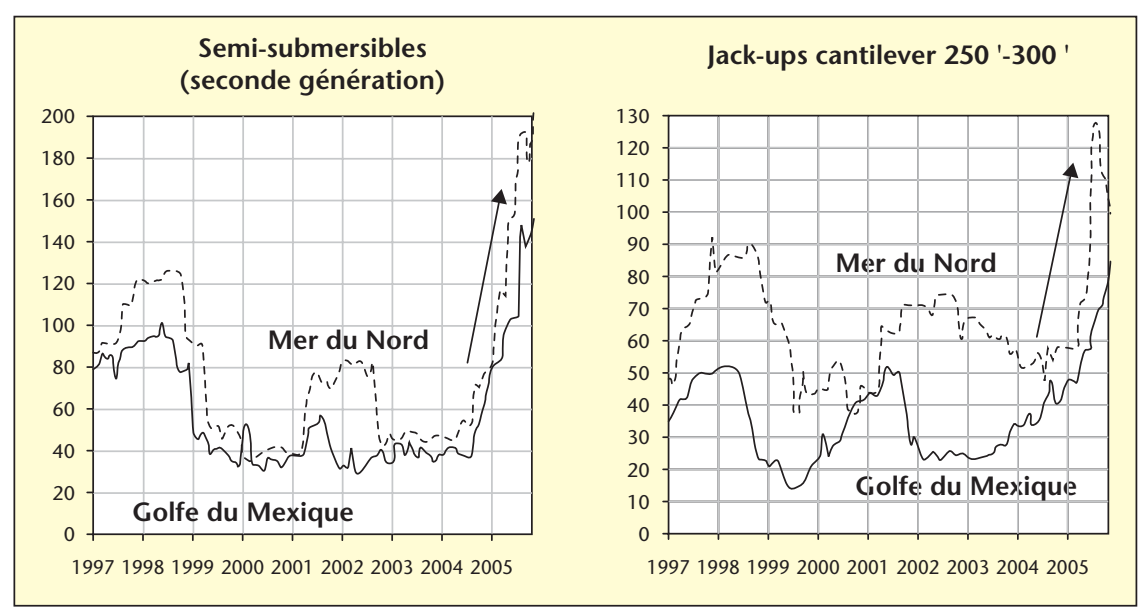

Figure 5. Taux de location des plates-formes de forage en mer (1 000 dollars US/jour). Source : Offshore Rig Locator.

pas qu'en 1998 une offre trop importante de brut sur le marché international avait entraîné une chute du prix du baril qui était descendu au-dessous de $15 \$$. Un prix du brut au-dessus de $50 \$ / b$ leur convient et les compagnies nationales des grands pays producteurs avancent donc prudemment dans la mise sur le marché de nouvelles capacités de production. Dans les deux à trois ans à venir, les volumes de production additionnels provenant des pays non Opep pourraient permettre de satisfaire la croissance de la demande, au-delà les choses sont plus incertaines.

Hors Opep, si certains pays, notamment du continent africain, voient leur production crô̂tre, d'autres grandes zones de production sont aujourd'hui en déclin, comme la mer du Nord.
L'argument de l'exploitation des ressources colossales de pétrole non conventionnelles est souvent mis en avant pour palier ces déclins. Le problème reste que l'exploitation de ces ressources se mettra en place lentement. Les exploiter signifie trouver, à un coût acceptable, les volumes importants d'énergie et d'eau nécessaires aux techniques de production qu'elles exigent. Sur la base des projets existants, à l'horizon 2015, ces pétroles devraient représenter une production additionnelle de $2,5 \mathrm{Mb} / \mathrm{j}$. À titre de comparaison, la croissance de la demande mondiale sur la période 20022004 a été de $4,6 \mathrm{Mb} / \mathrm{j}$, soit près de deux fois plus en seulement 3 ans (figure 6).

Le problème des capacités de production amène également à s'interroger sur le rythme

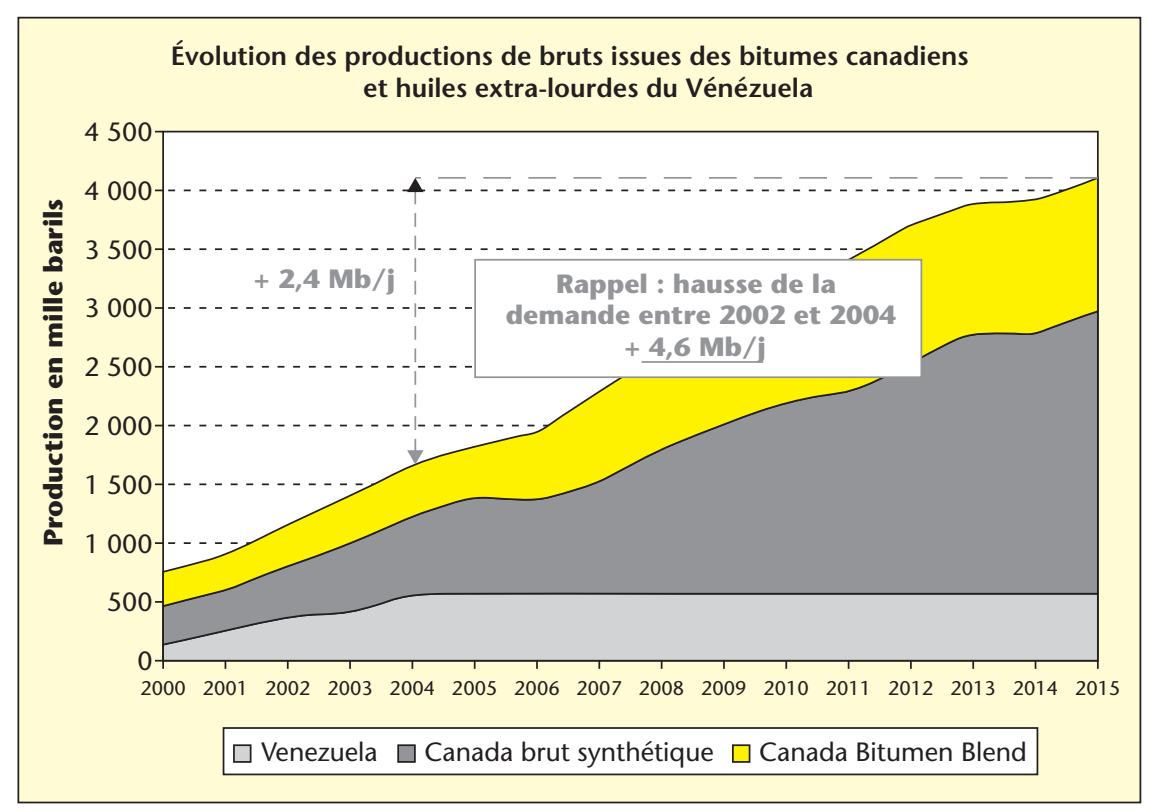

Figure 6. La contribution des ressources non conventionnelles à la production mondiale de pétrole. Source: IFP. 
de développement des capacités de production de brut de l'Opep et pour commencer à mettre en perspective les évolutions passées.

Hors effets de la guerre du Golfe (destructions/reconstructions), c'est-à-dire en excluant l'Iraq et le Koweït, les capacités de production des pays de l'Opep ont augmenté en moyenne de 0,7 à $0,8 \mathrm{Mb} / \mathrm{j}$ au début des années 90 (figure 7).

Le tassement de la demande et l'effondrement des prix en 1998 consécutif à la crise financière asiatique ont par la suite largement contribué à dissuader les pays exportateurs de se lancer dans des expansions de capacités. Les huit années qui ont suivi la crise asiatique ont vu une légère érosion des capacités qui contraste avec I'augmentation attendue en 2006 qui devrait être d'un million de barils par jour. S'agit-il d'un changement de rythme annonciateur de plusieurs années de hausses similaires à celles du début des années 90 ou bien d'un effort difficilement soutenable sur le long terme face au déclin naturel des champs du MoyenOrient?

L'évolution du prix du baril à court et moyen terme est également très fortement dépendant de l'évolution de la demande qui renvoie ellemême à plusieurs incertitudes dont le potentiel réel de développement des deux grands pays que sont la Chine et I'Inde et l'impact des prix en général (figure 8).

Bien que le concept de ratio de revenus et de consommation par habitant ne soit pas exempt de critiques sur certains points, nous avons situé le niveau actuel de développement de la Chine sur le sentier suivi par le Japon. II apparaît, sans perdre de vue les effets pervers associés à ce mode de calcul, qu'un Chinois dispose actuellement d'un revenu moyen (exprimé en dollar constant) équivalent à celui d'un Japonais dans les années 50 . La comparaison des consommations énergétiques est en cohérence avec la comparaison des revenus : la consommation pétrolière par habitant en Chine (inférieure à 2 barils par habitant et par an) est identique à celle du Japon à la même époque et la consommation d'électricité par habitant y est égale à celle du Japon en 1960 (environ $1000 \mathrm{kWh}$ par an).

On ne peut raisonnablement déduire de cette mise en perspective que la Chine suivra, au cours des prochaines décennies, un mode de développement identique, en rythme comme en nature, à celui du Japon. Mais le diagnostic que les besoins potentiels à satisfaire à l'échelle de l'individu sont très importants n'est pas contestable. La satisfaction de ces besoins à l'échelle de la population chinoise est ainsi susceptible d'engendrer de nouveau des répercussions profondes sur les marchés énergétiques comparables à celles de l'année 2004.

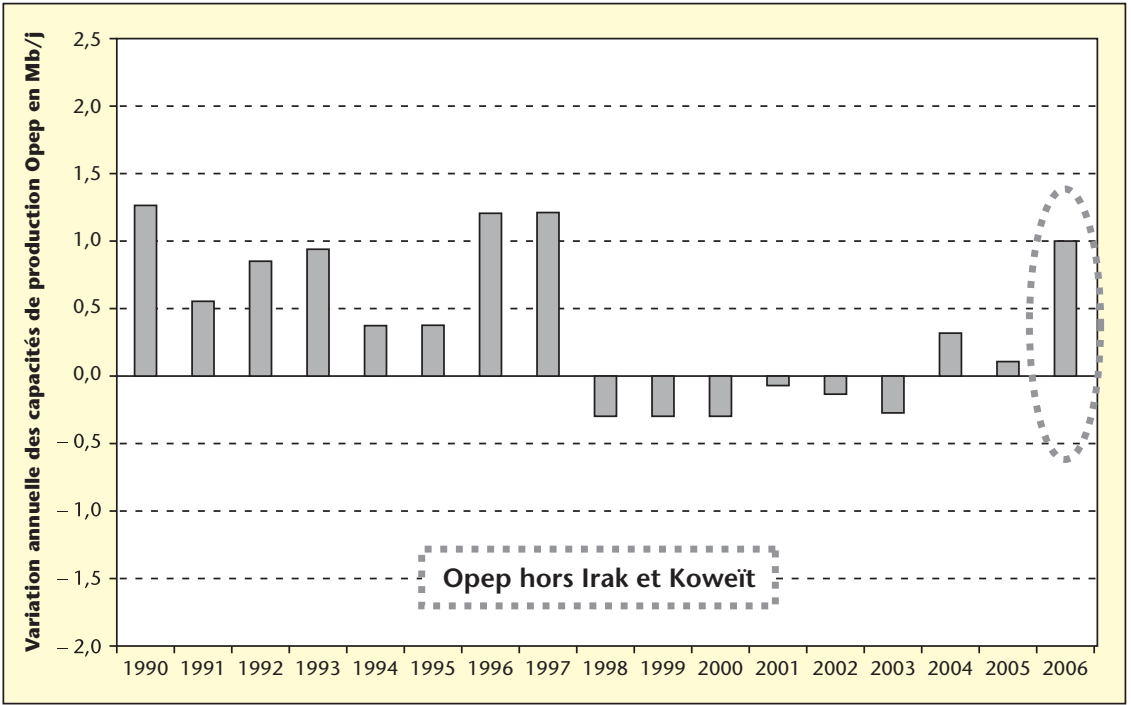

Figure 7. 2006 : vers une accélération du développement des capacités de l'Opep? Source: IFP.

L'évolution future de la demande renvoie également au problème de l'impact des prix en général.

II n'est pas aisé de synthétiser des éléments de réponse dans ce domaine car il existe une très grande diversité de régimes de prix de détails domestiques allant des pays ayant adopté une taxation lourde à ceux entretenant des subventions élevées, en passant par toute une palette de cas intermédiaires caractérisés par des niveaux de taxation ou de subvention modérés.

Le maintien d'un système d'encadrement des prix sur le marché domestique peut rapide-

ment, quand les prix sur les marchés internationaux flambent, devenir insoutenable pour le budget d'un état importateur net et amener celui-ci à abandonner un tel système. Ceci se traduit par une augmentation forte des prix des produits et à une réaction de la demande.

La Thaillande fournit un excellent exemple du rôle du régime de prix domestique dans l'évolution de la demande pétrolière. La mise en œuvre d'un système de subvention des prix des carburants, essence et gazole, au début de l'année 2004 a contribué à isoler le consommateur de la forte hausse des prix. La consomma-

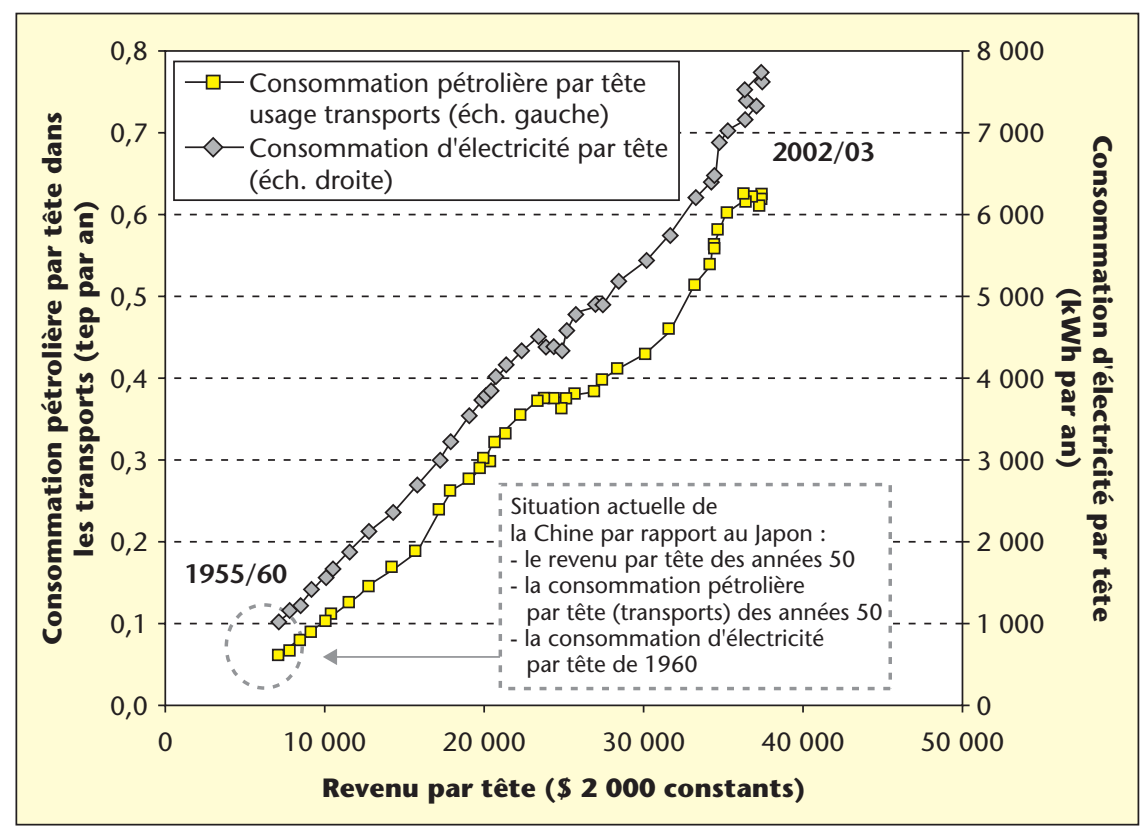

Figure 8. Les besoins potentiels de la Chine : niveau de développement comparable à celui du Japon en 1995. Source: IFP. 
tion totale d'essence a affiché une progression de $3 \%$ et celle de gazole de $10 \%$. Le coût très élevé induit par ce système a amené les autorités à éliminer la subvention sur l'essence à la fin de l'année 2004 mais à maintenir celle sur le gazole qui alimente essentiellement les usages commerciaux, c'est-à-dire le cœur de l'économie. Le résultat au cours de l'année 2005 a été spectaculaire : la consommation de gazole a conservé une forte croissance, quoiqu'en léger ralentissement, tandis que celle d'essence baissait de $6 \%$ environ sous l'effet de la transmission au consommateur des variations de prix internationaux (figure 9).

Les pays hors OCDE représentant $40 \%$ de la consommation mondiale mais surtout $75 \%$ de la croissance du marché, le cas de la Thaillande donne un aperçu, sans qu'il soit possible (dans l'immédiat) de le quantifier précisément, de l'impact potentiel sur la demande pétrolière si les principaux systèmes de subventions devaient être remis en cause. II s'agit d'un problème particulièrement sensible car il convient d'arbitrer, pour les pays concernés, entre le coût macro-économique croissant mais relativement indolore car différé des subventions et le coût macro-économique et social, voire politique, immédiat consécutif à la réduction des subventions.

Dans les 3 à 4 années à venir, si le rythme de la demande mondiale se maintient à un niveau de l'ordre de $1,5 \%$ à $2 \%$ d'accroissement, il est peu probable de voir se reconstituer les capacités excédentaires de production à leur niveau des années 90. Le prix du baril devrait donc rester élevé. II faudrait pour une reconstitution de ces capacités excédentaires de production que le rythme annuel d'accroissement de la demande mondiale tombe à des niveaux de $1,2 \%$ voire moins (figure 10 ).

À plus long terme, la réponse de la demande au prix pourrait être plus importante. L'heure est à l'accélération des mesures réglementaires et des politiques dans le domaine de l'énergie. On assiste aujourd'hui à une multiplication des plans d'actions nationaux en matière d'énergie qui visent tous à un accroissement de l'indépendance énergétique via la relance de programmes de maîtrise de la demande et le recours accru aux énergies nationales : c'est le retour en force du nucléaire, la volonté marquée des pays possédant des ressources de charbon de les valoriser (États-Unis, Australie, etc.).

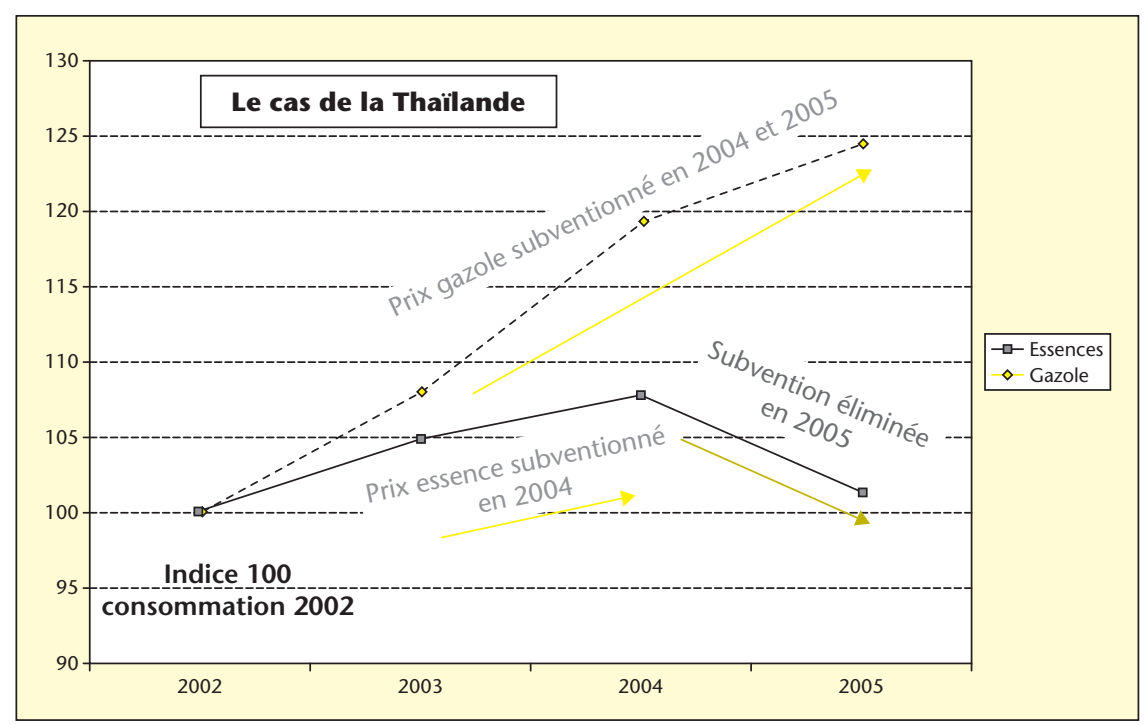

Figure 9. Le rôle des subventions dans le rythme de consommation pétrolière hors $O C D E$ : le cas de la Thailande. Source: IFP.

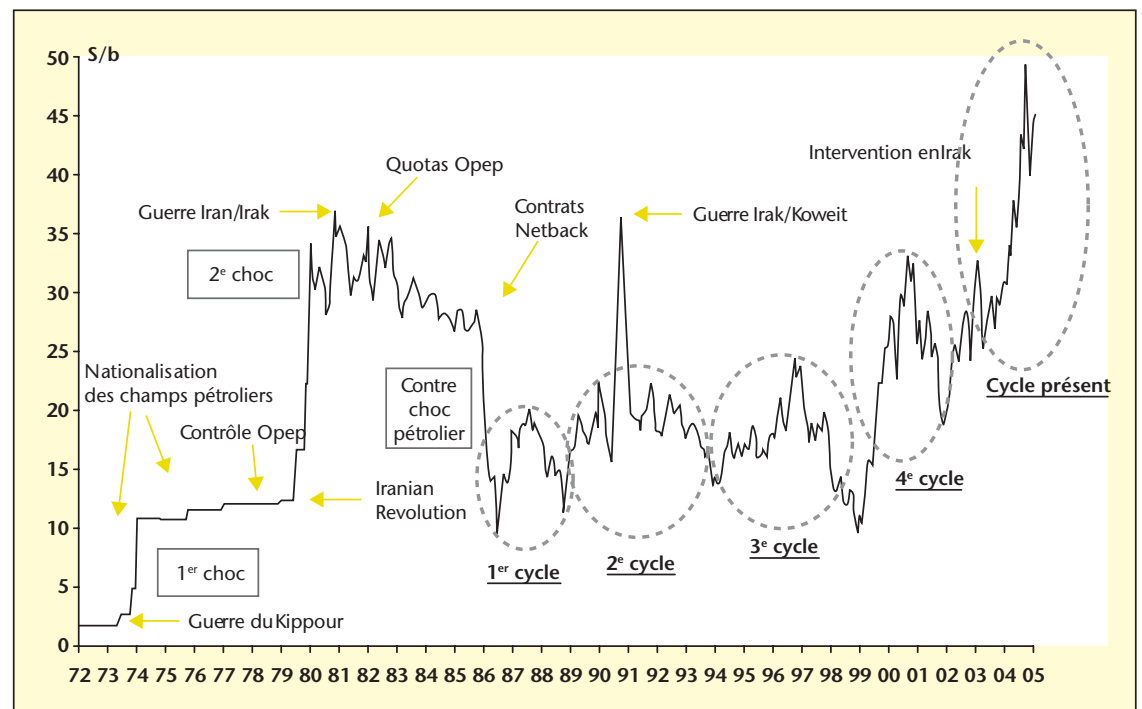

Figure 10. Le prix du pétrole un nouveau cycle?

Source: IFP.

Pour conclure, il apparaît aujourd'hui plus que probable que le prix du baril reste durablement élevé même si, comme tout marché de matière première, le marché pétrolier est un marché cyclique. Une baisse du prix du baril, découlant $d^{\prime}$ un tassement de la demande à court-moyen terme est envisageable, mais il paraît aujourd'hui peu probable sauf décélération de la demande que le prix du baril passe durablement au-dessous de $40 \$$. Le prix du baril de brut devrait donc rester élevé, favorisant le développement de solutions alternatives et notamment de carburants alternatifs comme les biocarburants. 